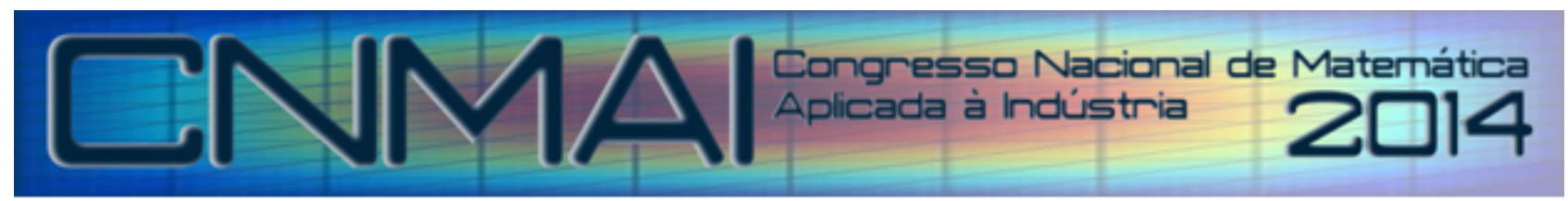

18 a 21 de novembro de 2014, Caldas Novas - Goiás

\title{
KINEMATIC MODELING OF A MULTI-FINGERED HAND PROSTHESIS FOR MANIPULATION TASKS
}

\author{
Marcelo H. Stoppa, mhstoppa@pq. cnpq. br ${ }^{1}$ \\ João Carlos Mendes Carvalho, jcmendes@mecanica.ufu.br ${ }^{2}$ \\ ${ }^{1}$ Universidade Federal de Goiás - Regional Catalão - Matemática Industrial \\ Av. Dr. Lamartine P. Avelar, 1120, St. Universitário - Catalão - GO - Brasil \\ ${ }^{2}$ Universidade Federal de Uberlândia - Faculdade de Engenharia Mecânica \\ Av. João Naves de Ávila, 2121, Sta. Mônica - Uberlândia - MG - Brasil
}

\begin{abstract}
Several different models represent the human hand. These describe hand models with up to 26 degrees of freedom (DoFs) which are needed to obtain an efficient representation of manipulation tasks by prosthesis. However, a great number of degrees of freedom leads to a complex control system and necessitate simplification. Consequently simplified models involve errors when is needed reconstruct hand's postures in daily tasks. This paper analyses a model proposed by InMoov Open Source project whose robotic hand has five fingers, able to perform palmar arc, in a total of 17 DoFs. Here is described a complete kinematic model to this artificial hand, where the model equations are calculated by means Denavit-Hartenberg coordinates. The model is able to perform just flexion joints movements, where the thumb, index and middle fingers have 3 links and 3 flexion joints. The ring and little fingers have 4 links and 4 flexion joints that permit simulation in palmar arc condition. After this some computational simulations are realized to compare the real model and the mathematical model under some simple grasping tasks.
\end{abstract}

Keywords: Kinematic, Prosthesis, Hand, Modelling, InMoov

\section{INTRODUCTION}

Human been has searched tirelessly to reproduce machines that can help or to substitute their actions. When a man loses a body part by some accident, end up being harmed their activities in different ways. If the hand is the body part which has been damaged, in general the problem becomes bigger. Accordingly, several research has been developed for the production of prostheses that approximate to the actual human hand.

Exist some commercial hand prosthesis such as Vincent (Vincent Systems), iLimb and iLimb Pulse (Touch Bionics), Bebionic (RSL Steeper) and Michelangelo (Otto Bock) (Smagt et al., 2009). However a great impediment is that hand prosthesis or robotics arms are very expensive (Allin et al., 2010).

Among the many works in the area of prosthetic hand, there are those that aimed to analyze the mechanical, others only the kinematics design, which can point out the following. Smagt et al. (2009) states that rather than to copy the intrinsic structure, in prosthesis design the goal is to closely copy the properties of the real human hand. Du e Charbon (2007) presents a 3D hand model fitting model which can recover finger positions with precision. Gosselin et al. (2008) presents an optimal geometry of tendon-driven fingers to an anthropomorphic under actuated robotic hand with 15 DoFs by a single actuator. Dalley et al. (2009) develops an anthropomorphic prototype hand prosthesis with 16 joints differentially driven by a set of five independent actuators where each joint finger have a torsional spring. Gaiser et al. (2009) makes a combination of flexible fluidic actuators and soft passive elements to reduce the required grasping force for several objects. Dechev et al. (2001) presents an experimental, multiple finger, prosthetic hand designed for children in the 7-11 year age group that results in a hand with reduced size and weight appropriated to kids. Baril et al. (2013) propose an anthropomorphic gripper for prosthetic applications which a mechanical lever is mounted inside the palm to per miss a proper distribution of the forces and provides mechanical advantage.

Specifically concerning the form, according Belter et al. (2013) the prosthesis design have to count the weight of entire system and extra charge to be carried by the user, so the weight is one of most important characteristics in its 
development. Anatomically, exists more or less independence between the fingers of the hand. In this way, Ingram et al. (2008) states that the thumb is the most independent of the digits and the ring finger is the most dependent one.

The inMoov project (Open Source), proposed by a modelmaker Gael Langevin (France), gathers efforts around the production of a full size humanoid robot and free offers to download by web, the design hands that can be printed by a $3 \mathrm{D}$ print. However in the opposite to costs of commercial prosthesis we search a project that can lead to a more cheap prosthesis. In this sense, this paper proposes a kinematic analysis and computational moves simulation of InMoov project's robotic hand for further adaptation and use it as prosthetic hand.

\section{KINEMATIC MODEL}

The prosthetic hand model analysed here is based on the human skeleton. However it has simplifications. While the real human hand model has over $20 \mathrm{DoFs}$, the model presented here is comprised 12 kinks that simulate the corresponding human bones, and 17 DoFs that represent the joints. The notation utilized to the model considers $\ell_{i, j}$ and $\theta_{i, j}$, respectively, to links and joints, where $i$ represents a finger and $j$ represents a joint or link corresponding as showed at Table 1 .

Table 1. Notation to joints and links in the model

\begin{tabular}{cccccc} 
& \multicolumn{5}{c}{ FINGERS } \\
\hline joints & Little (L) & Ring (R) & Middle (M) & Index (I) & Thumb (T) \\
\hline distal interphalangeal (DIP) & $\theta_{L, D I P}$ & $\theta_{R, D I P}$ & $\theta_{M, D I P}$ & $\theta_{I, D I P}$ & - \\
proximal interphalangeal (PIP) & $\theta_{L, P I P}$ & $\theta_{R, P I P}$ & $\theta_{M, P I P}$ & $\theta_{I, P I P}$ & - \\
metacarpophalangeal (MCP) & $\theta_{L, M C P}$ & $\theta_{R, M C P}$ & $\theta_{M, M C P}$ & $\theta_{I, M C P}$ & $\theta_{T, M C P}$ \\
carpometacarpal (CMC) & $\theta_{L, C M C}$ & $\theta_{R, C M C}$ & - & - & - \\
interphalangeal (IP) & - & - & - & - & $\theta_{T, I P}$ \\
trapeziometacarpal (TMC) & - & - & - & - & $\theta_{T, T M C}$ \\
\hline \hline links & Little (L) & Ring (R) & Middle (M) & Index (I) & Thumb (T) \\
\hline metacarpal (MC) & $\ell_{L, M C}$ & $\ell_{R, M C}$ & - & - & $\ell_{T, M C}$ \\
proximal (P) & $\ell_{L, P}$ & $\ell_{R, P}$ & $\ell_{M, P}$ & $\ell_{I, P}$ & $\ell_{T, P}$ \\
middle (M) & $\ell_{L, M}$ & $\ell_{R, M}$ & $\ell_{M, M}$ & $\ell_{I, M}$ & - \\
distal (D) & $\ell_{L, D}$ & $\ell_{R, D}$ & $\ell_{M, D}$ & $\ell_{I, D}$ & $\ell_{T, D}$ \\
\hline
\end{tabular}

Therefore, assuming this configuration, the thumb (T), index (I), and middle (M) fingers have 3 joints and 3 links each while ring (R) and little (L) fingers have 4 joints and 4 links each, a total of 17 degrees of freedom (DoF). The $\theta_{L, C M C}$ and $\theta_{R, C M C}$ DoFs allows to simulate the palm arc, a kind of deformation that occurs when the prosthetic hand is grasping a ball or any similar object like this.

\subsection{Denavit-Hartenberg Convention}

When we are considering a reduced number of DoFs in a prosthesis hand design, is needed to improve the project for reach a better handling of objects an shapes in a effective way. So, the mechanical design is an important aspect that need special attention where simulation can be helpful at overall. Mathematical modelling is a first step to establish a good simulation. The following is described the mathematical modelling comprising the kinematics aspects from prosthesis, that is the study of motion without taking into account what causes the motion (Tarzimi et al., 2009). Model equations are calculated by means of Denavit-Hartenberg modified convention (Khalil e Kleinfinger, 1986) where the frames receive labels according to which joint they are positioned. Let us consider the following:

- the $Z$ axis of a generic frame, namely $\{i\}$, is called $Z_{i}$, coincident with the joint $i$;

- the origin of $\{i\}$ is situated where the $\alpha_{i}$ perpendicularly intersects the joint $i$ axis;

- the parameters wich lead to define frame $\{i\}$ will have $(i)$ as subscript;

- joint $(i)$ connects links $i-1$ and $i$;

- $X_{i}$ is the common perpendicular between $Z_{i}$ and $Z_{i+1}$.

Here we are considering just flexion movements and 3 different kinematic configurations:

I) For the thumb, with 3 joints and 3 links where the trapeziometacarpal joint have a frame whose axis are not mutually parallel to metacarpophalangeal and interphalangeal joints (Fig. 1).

II) For the index and middle fingers $(i=I, M)$, also with 3 joints and 3 links all frames axis are mutually parallel (Fig.2)

III) For the ring and little fingers $(i=R, L)$, with 4 joints and 4 links, the carpometacarpal frame $\{C M C\}$ has axes not mutually parallel to the other frames (Fig. 3).

The Fig. 4 shows the complete model with 5 fingers, 17 DoFs and a general frame is positioned in the wrist and the vectors $u_{L}, u_{R}, u_{M}, u_{I}$ and $u_{T}$ relate the initial frames at each finger with the central wrist referential. 


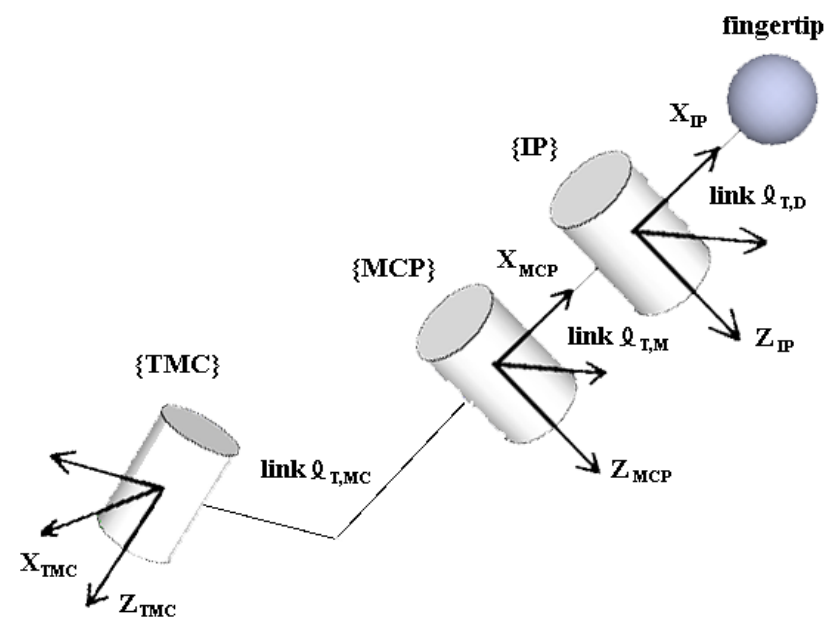

Figure 1. Model for the thumb

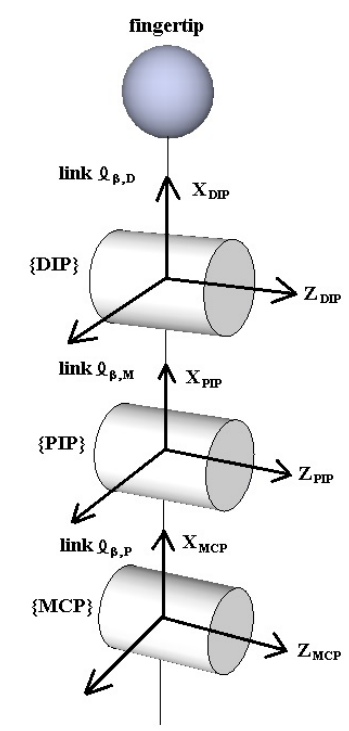

Figure 2. Model for the index and middle fingers

\subsection{Forward Kinematic}

The forward kinematic solution of each finger will be assigned using homogeneous matrices and is used to determine the position and orientation of fingertips relative to the base coordinate system located in the center of wrist. Model equations are calculated by means of Denavit-Hartenberg (D-H) parameters where human anatomical terminology has been used to describe the prosthesis model. Considering the 3 configurations as in the previous section, the D-H parameters are determined according modified convention proposed by Khalil e Kleinfinger (1986). The variables of the model, named $\theta_{i, j}$, represent the flexion angle in each joint. In all D-H parameters $i$ and $j$ indices represents, respectively, the corresponding finger and joint. After setting frames according to the D-H convention, the parameters $\theta_{i, j}, d_{i, j}, \alpha_{i, j}$ and $r_{i, j}$ are such that:

- $\theta_{i}$ is the angle between $X_{i-1}$ and $X_{i-1}$ about $Z_{i}$ axis;

- $d_{i}$ is the distance between origin $O_{i-1}$ and $X_{i-1}$ axis;

- $\alpha_{i}$ is the angle between $Z_{i-1}$ and $Z_{i-1}$ about $X_{i-1}$ axis;

- $r_{i}$ is the distance between origin $O_{i}$ and $X_{i-1}$ axis.

To compute the fingertip position is needed to consider an additional joint whose flexion angle equals zero.

\subsubsection{Kinematic of the thumb finger}

The forward kinematic for the thumb finger is defined according to the parameters of Table 2, as showed in Eq. (1).

$$
P_{T}={ }_{0}^{w} T_{T}\left(r_{T, w}\right){ }_{1}^{0} T_{T}\left(\theta_{T, T M C}\right){ }_{2}^{1} T_{T}\left(\theta_{T, M C P}\right){ }_{3}^{2} T_{T}\left(\theta_{T, I P}\right){ }_{4}^{3} T_{T}\left(\theta_{T, D}\right)
$$




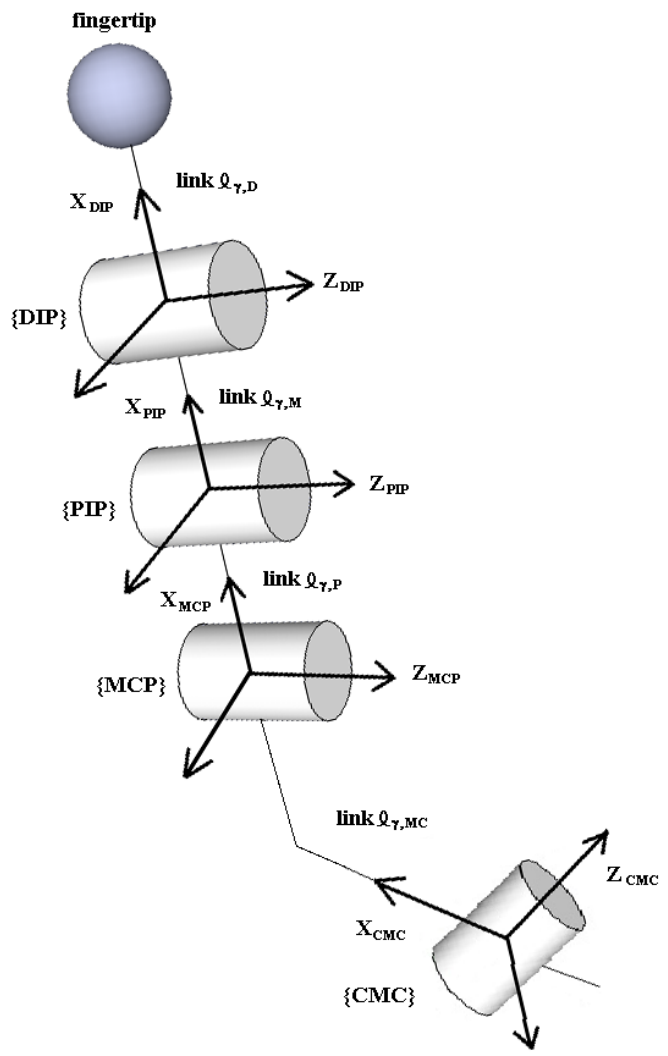

Figure 3. Model for the ring and little fingers

Table 2. D-H parameters for the thumb

\begin{tabular}{ccccc}
\hline joint & $\theta_{i, j}$ & $d_{i, j}$ & $r_{i, j}$ & $\alpha_{i, j}$ \\
\hline $\mathrm{w}$ & 0 & 0 & $r_{T, w}$ & 0 \\
1 & $\theta_{T, T M C}$ & 0 & $r_{T, M C}$ & $\alpha_{T, M C}$ \\
2 & $\theta_{T, M C P}$ & 0 & $r_{T, P}$ & $\alpha_{T, P}$ \\
3 & $\theta_{T, I P}$ & $d_{T, P}$ & 0 & 0 \\
4 & 0 & $d_{T, D}$ & 0 & 0 \\
\hline
\end{tabular}

where $P_{T}$ represents a matrix that contains position and orientation of the thumb tip with respect to the center of the wrist; $T_{T}$ are the matrices that represent the translation or rotation contribution of each joint, given in a general form (Craig, 2004) by:

$$
{ }_{k}^{k-1} T_{T}\left(\theta_{i, j}\right)=\left[\begin{array}{cccc}
\cos \left(\theta_{i, j}\right) & -\sin \left(\theta_{i, j}\right) & 0 & d_{i, j} \\
\cos \left(\alpha_{i, j}\right) \sin \left(\theta_{i, j}\right) & \cos \left(\alpha_{i, j}\right) \cos \left(\theta_{i, j}\right) & -\sin \left(\alpha_{i, j}\right) & -r_{i, j} \sin \left(\alpha_{i, j}\right) \\
\sin \left(\alpha_{i, j}\right) \sin \left(\theta_{i, j}\right) & \sin \left(\alpha_{i, j}\right) \cos \left(\theta_{i, j}\right) & \cos \left(\alpha_{i, j}\right) & r_{i, j} \cos \left(\alpha_{i, j}\right) \\
0 & 0 & 0 & 1
\end{array}\right]
$$

where $0-1=w$ representing the center of the wrist.

So, each of the matrices $T_{T}$ are given by:

$$
\begin{gathered}
{ }_{0}^{w} T_{T}\left(r_{T, w}\right)=\left[\begin{array}{cccc}
1 & 0 & 0 & 0 \\
0 & 1 & 0 & 0 \\
0 & 0 & 1 & r_{T, w} \\
0 & 0 & 0 & 1
\end{array}\right] \\
{ }_{s}^{s-1} T_{T}\left(\theta_{T, j}\right)=\left[\begin{array}{cccc}
\cos \left(\theta_{T, j}\right) & -\sin \left(\theta_{T, j}\right) & 0 & 0 \\
\cos \left(\alpha_{T, v}\right) \sin \left(\theta_{T, j}\right) & \cos \left(\alpha_{T, v}\right) \cos \left(\theta_{T, j}\right) & -\sin \left(\alpha_{T, v}\right) & -r_{T, v} \sin \left(\alpha_{T, v}\right) \\
\sin \left(\alpha_{T, v}\right) \sin \left(\theta_{T, j}\right) & \sin \left(\alpha_{T, v}\right) \cos \left(\theta_{T, j}\right) & \cos \left(\alpha_{T, v}\right) & r_{T, v} \cos \left(\alpha_{T, v}\right) \\
0 & 0 & 0 & 1
\end{array}\right]
\end{gathered}
$$




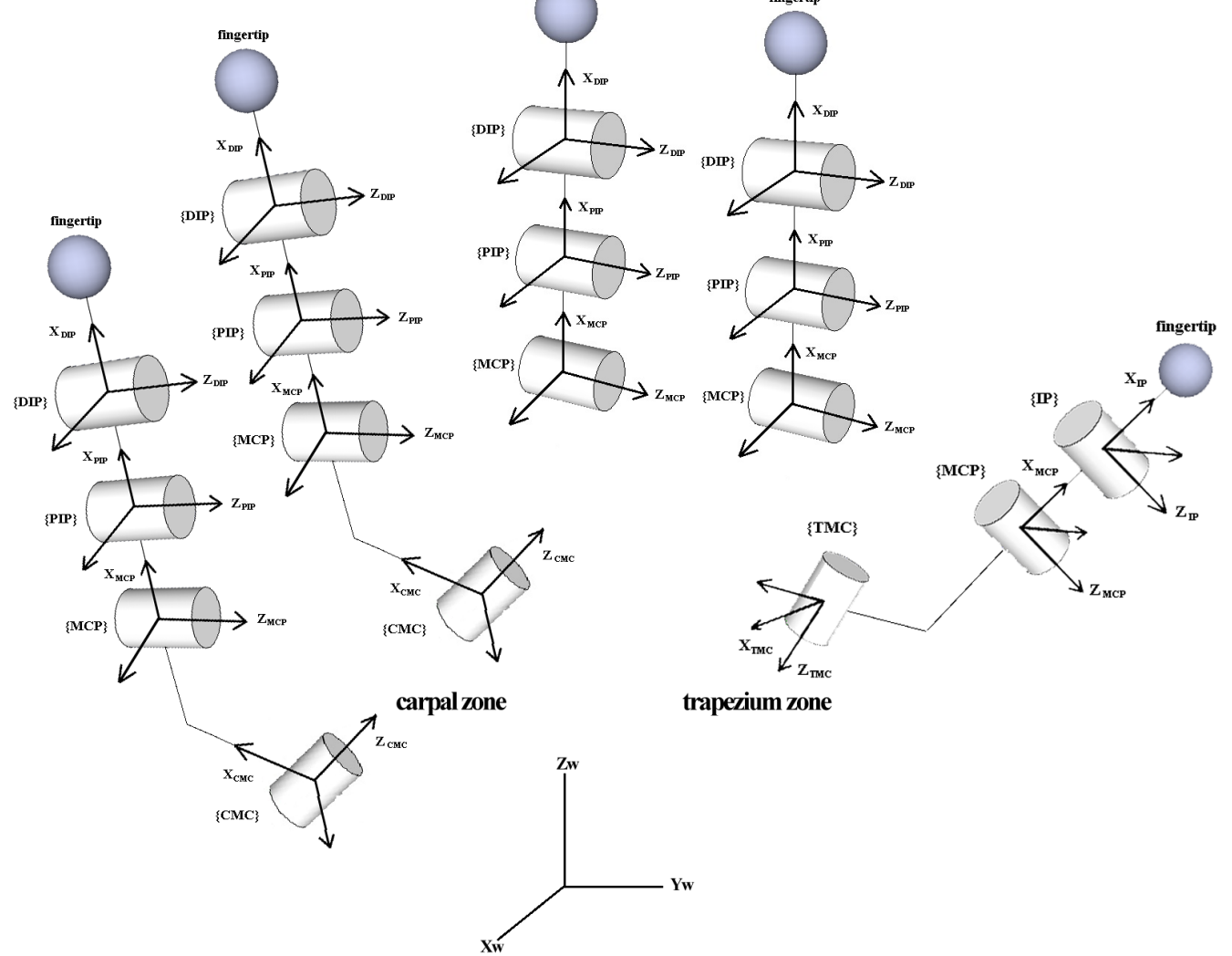

Figure 4. Complete kinematic configuration prosthesis

where $s=1,2, v=M C, P$ and $j=T M C, M C P$, in this order.

$$
\begin{aligned}
& { }_{3}^{2} T_{T}\left(\theta_{T, I P}\right)=\left[\begin{array}{cccc}
\cos \left(\theta_{T, I P}\right) & -\sin \left(\theta_{T, I P}\right) & 0 & d_{T, P} \\
\sin \left(\theta_{T, I P}\right) & \cos \left(\theta_{T, I P}\right) & 0 & 0 \\
0 & 0 & 1 & 0 \\
0 & 0 & 0 & 1
\end{array}\right] \\
& { }_{4}^{3} T_{T}\left(\theta_{T, D}\right)=\left[\begin{array}{cccc}
1 & 0 & 0 & d_{T, D} \\
0 & 1 & 0 & 0 \\
0 & 0 & 1 & 0 \\
0 & 0 & 0 & 1
\end{array}\right]
\end{aligned}
$$

\subsubsection{Kinematics of the index and middle fingers}

Similarly to the previous section, the forward kinematics for the index and middle fingers are defined according to the parameters of Table 3, as showed in Eq. (7) where $\beta=I, M$ (Index, Middle).

Table 3. D-H parameters for the index and middle fingers

\begin{tabular}{ccccc}
\hline joint & $\theta_{i, j}$ & $d_{i, j}$ & $r_{i, j}$ & $\alpha_{i, j}$ \\
\hline $\mathrm{w}$ & 0 & 0 & $r_{\beta, w}$ & 0 \\
1 & $\theta_{\beta, M C P}$ & 0 & $r_{\beta, M C}$ & $\alpha_{\beta, M C}$ \\
2 & $\theta_{\beta, P I P}$ & $d_{\beta, P}$ & 0 & 0 \\
3 & $\theta_{\beta, D I P}$ & $d_{\beta, M}$ & 0 & 0 \\
4 & 0 & $d_{\beta, D}$ & 0 & 0 \\
\hline
\end{tabular}

$$
P_{\beta}={ }_{0}^{w} T_{\beta}\left(r_{\beta, w}\right){ }_{1}^{0} T_{\beta}\left(\theta_{\beta, M C P}\right){ }_{2}^{1} T_{\beta}\left(\theta_{\beta, P I P}\right){ }_{3}^{2} T_{\beta}\left(\theta_{\beta, D I P}\right){ }_{4}^{3} T_{\beta}\left(\theta_{\beta, D}\right)
$$


where $P_{\beta}$ represents matrices that contains position and orientation of index and middle fingertips with respect to the center of the wrist; $T_{\beta}$ are the matrices like that showed in Eq. (2), given by:

$$
\begin{aligned}
& { }_{0}^{w} T_{\beta}\left(r_{\beta, w}\right)=\left[\begin{array}{cccc}
1 & 0 & 0 & 0 \\
0 & 1 & 0 & 0 \\
0 & 0 & 1 & r_{\beta, w} \\
0 & 0 & 0 & 1
\end{array}\right]
\end{aligned}
$$

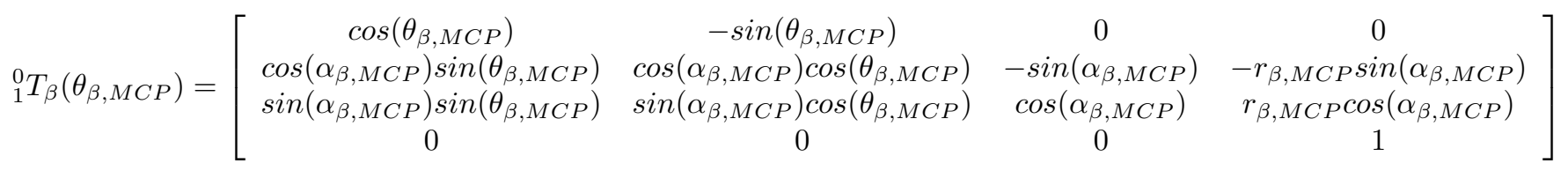

$$
\begin{aligned}
& { }_{s}^{s-1} T_{\beta}\left(\theta_{\beta, j}\right)=\left[\begin{array}{cccc}
\cos \left(\theta_{\beta, j}\right) & -\sin \left(\theta_{\beta, j}\right) & 0 & d_{\beta, v} \\
\sin \left(\theta_{\beta, j}\right) & \cos \left(\theta_{\beta, j}\right) & 0 & 0 \\
0 & 0 & 1 & 0 \\
0 & 0 & 0 & 1
\end{array}\right]
\end{aligned}
$$

where $s=2,3, j=P I P, D I P$ and $v=P, M$, in this order.

$$
{ }_{4}^{3} T_{\beta}\left(\theta_{\beta, D}\right)=\left[\begin{array}{cccc}
1 & 0 & 0 & d_{\beta, D} \\
0 & 1 & 0 & 0 \\
0 & 0 & 1 & 0 \\
0 & 0 & 0 & 1
\end{array}\right]
$$

\subsubsection{Kinematics of the ring and little fingers}

To the ring and little fingers, the forward kinematics are defined according to the parameters of Table 4, remembering that to this configuration we have 4 joints and 4 links. Equation (12) gives the matrices that contain position and orientation of ring and little fingertips with respect to the center of the wrist, where $\gamma=R, L$ (Ring, Little).

Table 4. D-H parameters for the ring and little fingers

$$
\begin{aligned}
& \begin{array}{ccccc}
\hline \text { joint } & \theta_{i, j} & d_{i, j} & r_{i, j} & \alpha_{i, j} \\
\hline \mathrm{w} & 0 & 0 & r_{\gamma, w} & 0 \\
1 & \theta_{\gamma, C M C} & 0 & r_{\gamma, M C} & \alpha_{\gamma, M C} \\
2 & \theta_{\gamma, M C P} & 0 & r_{\gamma, P} & \alpha_{\gamma, P} \\
3 & \theta_{\gamma, P I P} & d_{\gamma, P} & 0 & 0 \\
4 & \theta_{\gamma, D I P} & d_{\gamma, M} & 0 & 0 \\
5 & 0 & d_{\gamma, D} & 0 & 0 \\
\hline
\end{array} \\
& P_{\gamma}={ }_{0}^{w} T_{\gamma}\left(r_{\gamma, w}\right){ }_{1}^{0} T_{\gamma}\left(\theta_{\gamma, C M C}\right){ }_{2}^{1} T_{\gamma}\left(\theta_{\gamma, M C P}\right){ }_{3}^{2} T_{\gamma}\left(\theta_{\gamma, P I P}\right){ }_{4}^{3} T_{\gamma}\left(\theta_{\gamma, D I P}\right){ }_{5}^{4} T_{\gamma}\left(\theta_{\gamma, D}\right) \\
& { }_{0}^{w} T_{\gamma}\left(r_{\gamma, w}\right)=\left[\begin{array}{cccc}
1 & 0 & 0 & 0 \\
0 & 1 & 0 & 0 \\
0 & 0 & 1 & r_{\gamma, w} \\
0 & 0 & 0 & 1
\end{array}\right] \\
& { }_{s}^{s-1} T_{\gamma}\left(\theta_{\gamma, j}\right)=\left[\begin{array}{cccc}
\cos \left(\theta_{\gamma, j}\right) & -\sin \left(\theta_{\gamma, j}\right) & 0 & 0 \\
\cos \left(\alpha_{\gamma, v}\right) \sin \left(\theta_{\gamma, j}\right) & \cos \left(\alpha_{\gamma, v}\right) \cos \left(\theta_{\gamma, j}\right) & -\sin \left(\alpha_{\gamma, v}\right) & -r_{\gamma, v} \sin \left(\alpha_{\gamma, v}\right) \\
\sin \left(\alpha_{\gamma, v}\right) \sin \left(\theta_{\gamma, j}\right) & \sin \left(\alpha_{\gamma, v}\right) \cos \left(\theta_{\gamma, j}\right) & \cos \left(\alpha_{\gamma, v}\right) & r_{\gamma, v} \cos \left(\alpha_{\gamma, v}\right) \\
0 & 0 & 0 & 1
\end{array}\right]
\end{aligned}
$$


where $s=1,2, v=M C, P$ and $j=C M C, M C P$, in this order.

$$
{ }_{h}^{h-1} T_{\gamma}\left(\theta_{\gamma, k}\right)=\left[\begin{array}{cccc}
\cos \left(\theta_{\gamma, k}\right) & -\sin \left(\theta_{\gamma, k}\right) & 0 & d_{\gamma, w} \\
\sin \left(\theta_{\gamma, k}\right) & \cos \left(\theta_{\gamma, k}\right) & 0 & 0 \\
0 & 0 & 1 & 0 \\
0 & 0 & 0 & 1
\end{array}\right]
$$

where $h=1,2, w=P, M$ and $k=P I P, D I P$, in this order.

$$
{ }_{5}^{4} T_{\gamma}\left(\theta_{\gamma, D}\right)=\left[\begin{array}{cccc}
1 & 0 & 0 & d_{\gamma, D} \\
0 & 1 & 0 & 0 \\
0 & 0 & 1 & 0 \\
0 & 0 & 0 & 1
\end{array}\right]
$$

\section{RESULTS}

With the mathematical model, it is possible to implement the equations of motion and simulate some grasping situations of the prosthesis. Actual measurements of the prosthesis are used as model parameters, which can be observed in the Table 5.

\begin{tabular}{|c|c|c|c|c|c|c|c|}
\hline \multicolumn{8}{|c|}{ thumb } \\
\hline \multicolumn{2}{|c|}{$\theta_{i, j}$} & \multicolumn{2}{|c|}{$d_{i, j}$} & \multicolumn{2}{|c|}{$r_{i, j}$} & \multicolumn{2}{|c|}{$\alpha_{i, j}$} \\
\hline$\theta_{T, T M C}$ & 0 to 40 & & & $r_{T, w}$ & -304.5206 & & \\
\hline$\theta_{T, M C P}$ & 0 to 60 & $d_{T, P}$ & 37 & $R_{T, M C}$ & -383.8076 & $\alpha_{T, M C}$ & 3.0466 \\
\hline$\theta_{T, I P}$ & 0 to 60 & $d_{T, D}$ & 32 & $R_{T, P}$ & 42.4336 & $\alpha_{T, P}$ & 0.7321 \\
\hline \multicolumn{8}{|c|}{ index } \\
\hline \multicolumn{2}{|c|}{$\theta_{i, j}$} & \multicolumn{2}{|c|}{$d_{i, j}$} & \multicolumn{2}{|c|}{$r_{i, j}$} & \multicolumn{2}{|c|}{$\alpha_{i, j}$} \\
\hline$\theta_{I, M C P}$ & 0 to 100 & $d_{I, P}$ & 38 & $r_{I, w}$ & 92 & & \\
\hline$\theta_{I, P I P}$ & 0 to 80 & $d_{I, M}$ & 24 & $r_{I, M C}$ & 40.1528 & $\alpha_{I, M C}$ & -1.6581 \\
\hline$\theta_{I, D I P}$ & 0 to 60 & $d_{I, D}$ & 30.5 & & & & \\
\hline \multicolumn{8}{|c|}{ middle } \\
\hline \multicolumn{2}{|c|}{$\theta_{i, j}$} & \multicolumn{2}{|c|}{$d_{i, j}$} & \multicolumn{2}{|c|}{$r_{i, j}$} & \multicolumn{2}{|c|}{$\alpha_{i, j}$} \\
\hline$\theta_{M, M C P}$ & 0 to 100 & $d_{M, P}$ & 41 & \multirow{3}{*}{$\begin{array}{c}r_{M, w} \\
r_{M, M C}\end{array}$} & \multirow{3}{*}{$\begin{array}{c}96 \\
13.0317\end{array}$} & & \multirow{3}{*}{-1.6406} \\
\hline$\theta_{M, P I P}$ & 0 to 80 & $d_{M, M}$ & 27 & & & $\alpha_{M, M C}$ & \\
\hline$\theta_{M, D I P}$ & 0 to 60 & $d_{M, D}$ & 34 & & & & \\
\hline \multicolumn{8}{|c|}{ ring } \\
\hline \multicolumn{2}{|c|}{$\theta_{i, j}$} & \multicolumn{2}{|c|}{$d_{i, j}$} & \multicolumn{2}{|c|}{$r_{i, j}$} & \multicolumn{2}{|c|}{$\alpha_{i, j}$} \\
\hline$\theta_{R, C M C}$ & 0 to 25 & & & $r_{R, w}$ & 52.8391 & & \\
\hline$\theta_{R, M C P}$ & 0 to 80 & $d_{R, P}$ & 33 & $r_{R, M C}$ & 65.1065 & $\alpha_{R, M C}$ & -0.8727 \\
\hline$\theta_{R, P I P}$ & 0 to 80 & $d_{R, M}$ & 22 & $r_{R, P}$ & -65.4793 & $\alpha_{R, P}$ & -0.6028 \\
\hline$\theta_{R, D I P}$ & 0 to 70 & $d_{R, D}$ & 28 & & & & \\
\hline \multicolumn{8}{|c|}{ little } \\
\hline \multicolumn{2}{|c|}{$\theta_{i, j}$} & \multicolumn{2}{|c|}{$d_{i, j}$} & \multicolumn{2}{|c|}{$r_{i, j}$} & \multicolumn{2}{|c|}{$\alpha_{i, j}$} \\
\hline$\theta_{L, C M C}$ & 0 to 25 & & & $r_{L, w}$ & 55.4641 & & \\
\hline$\theta_{L, M C P}$ & 0 to 80 & $d_{L, P}$ & 31 & $r_{L, M C}$ & 49.0825 & $\alpha_{L, M C}$ & -0.6778 \\
\hline$\theta_{L, P I P}$ & 0 to 80 & $d_{L, M}$ & 19 & $r_{L, P}$ & -74.5525 & $\alpha_{L, M C}$ & -0.6480 \\
\hline$\theta_{L, D I P}$ & 0 to 70 & $d_{L, D}$ & 25 & & & & \\
\hline
\end{tabular}

Table 5. Prosthesis parameters

Measurements were taken in milimeters and the angles in degrees. The origin of the coordinate system is positioned on the center line of the wrist base where the $x y$ plane is considered as being the midplane of the prosthesis.

Under the taxonomy proposed by Cutkosky (1989), the grasps are divided into power grasps and precision grasps. In the precision grasps the sensitivity and dexterity are predominant while the power grasps have large areas of contact between the grasped object and the surfaces of the fingers and palm, with little or no ability needed to perform movements.

Figure (5) exhibits the prosthesis with no flexion in the joints $\left(\theta_{i, j}=0\right.$ to all $\left.i, j\right)$ and a simulation for this situation under the implemented model. 

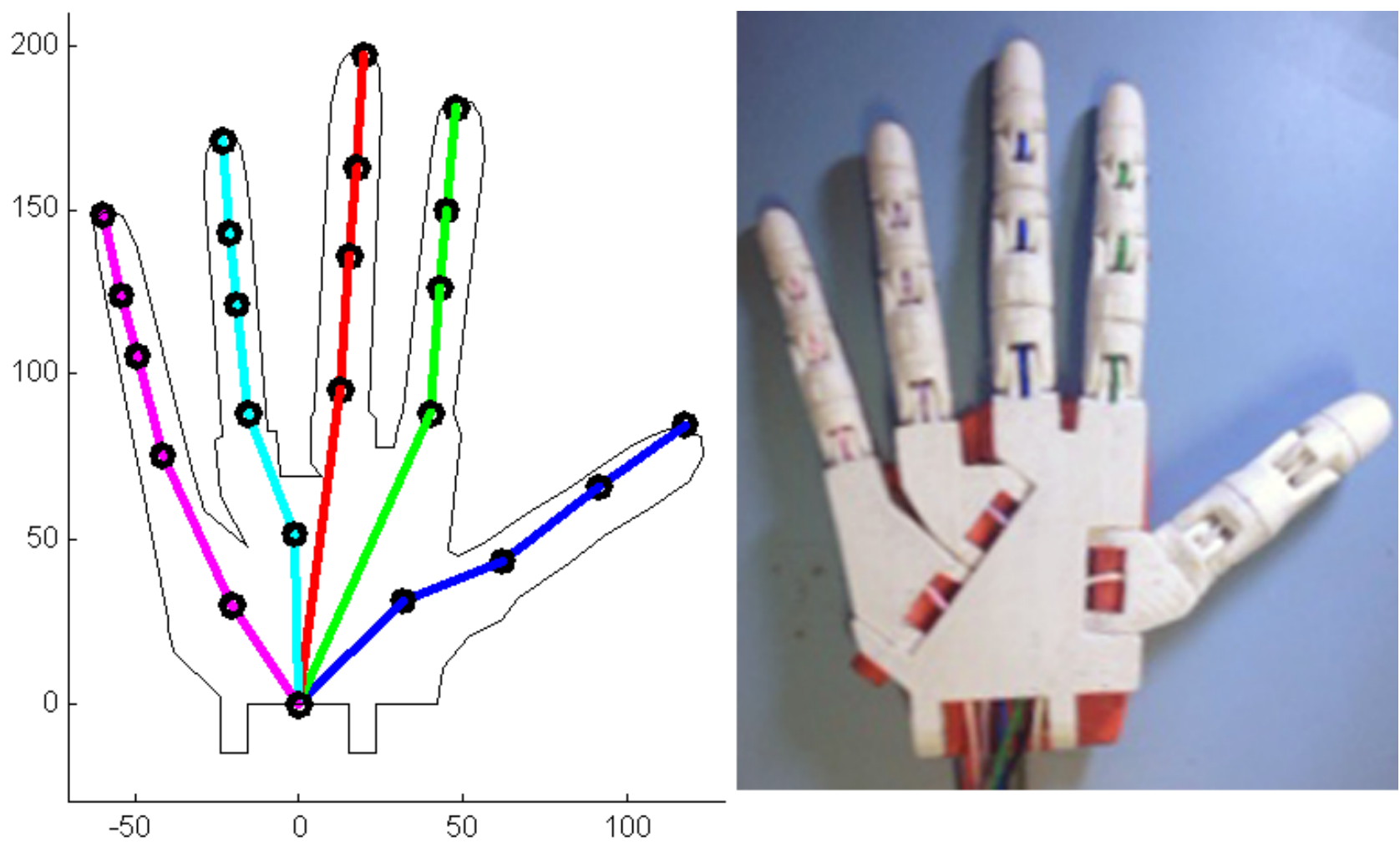

Figure 5. Simulated model and prosthesis with no flexion

Figure (6) presents a situation when index and thumb fingers are totally flexioned over his joints. We can perceive that exists a geometric difficulty to perform precision grasps, principally with thumb and index finger. Here is not considered analysis of contact between the fingers.

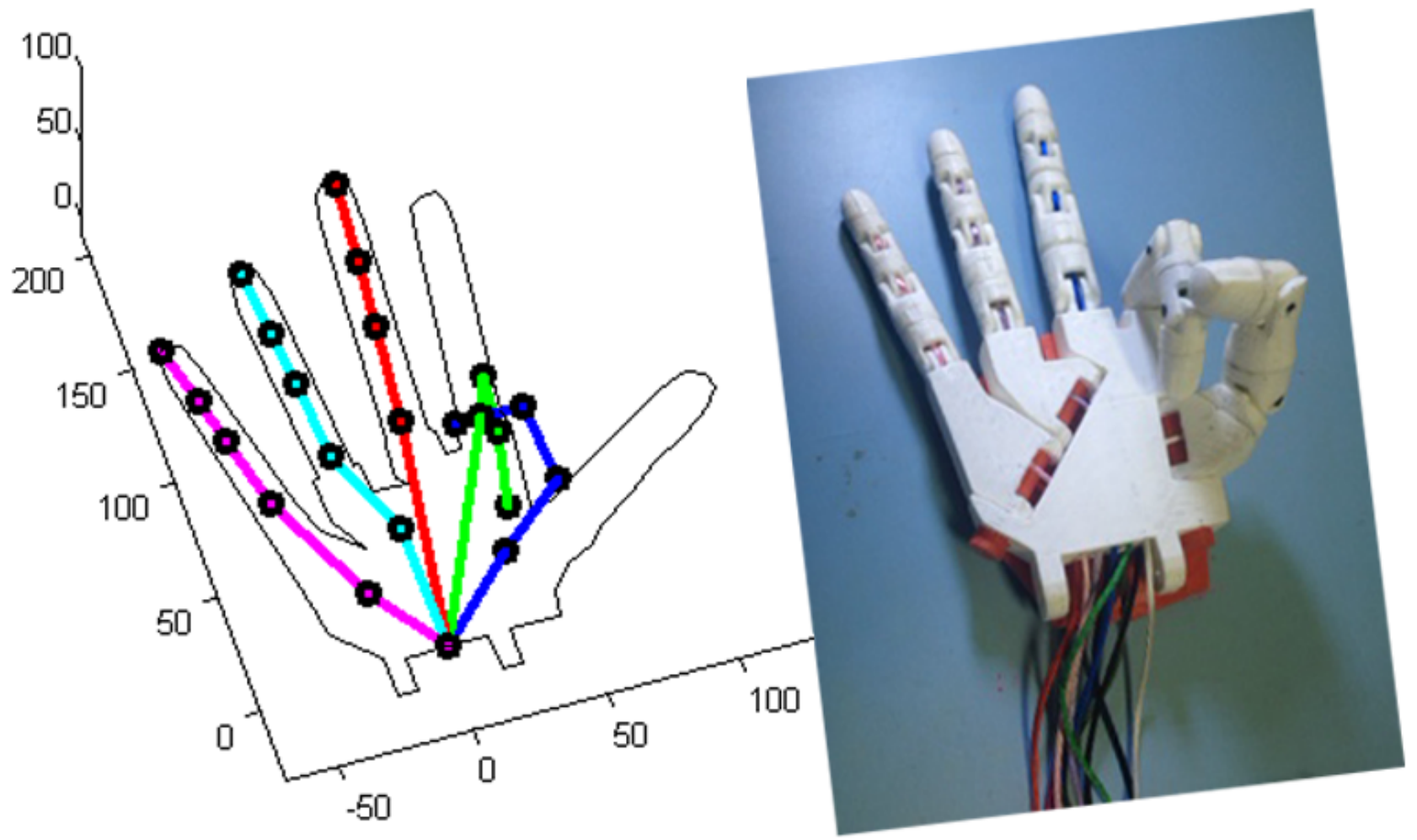

Figure 6. Simulated model and prosthesiswith thumb and index fully flexioned

Finally, Fig. (7) shows the prosthesis completely flexioned (maximal angles to each joint) and his corresponding modeled situation. 


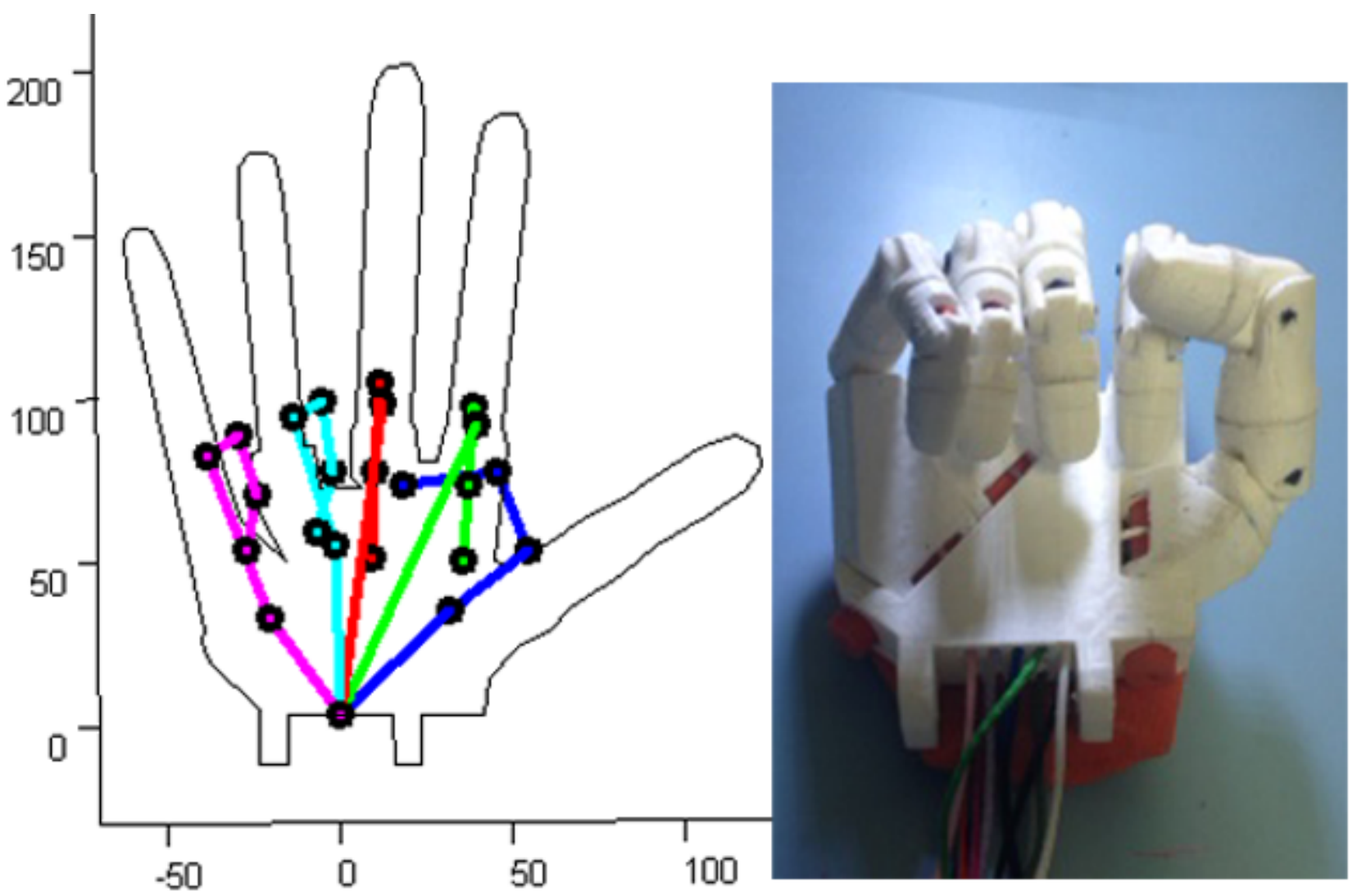

Figure 7. Simulated model and prosthesis completely flexioned

\section{CONCLUSION}

According Belter et al. (2013) the relationship between the rotation axis of the thumb and the main axis of the wrist is a critical parameter since it is fundamental to trajectory thumb and consequently to perform a lot of grasps. In this prosthesis hand analyzed here, the thumb rotation axis and index longitudinal axis are the same, which is a trouble to produce some kind of grasps. The thumb accounts for $40 \%$ of the entire functionality of the human hand. Therefore, it is clear that the placement of the thumb must be calculated to allow greater possibility of grasps.

In this paper we present a robotic hand developed into InMoov Open Source project (Langevin, 2014), compound by 5 fingers and a total of 17 DoFs. We develop a complete kinematic model to the robotic hand, where the equations are determined by Denavit-Hartenberg coordinates. There is a slight difference between the simulated model and the real, because of a slight twist of the thumb to the center of the hand, which was not considered in the simulation. With the model it was possible simulate some kind of common grasps and to compare with the real position of the fingers.

To use this InMoov robotic hand how prosthesis, several adjusts need to be made increasing the mobility and user freedom. Owing to possible errors in the hand's dimensions, the real model and the mathematical model show some differences. However, the computational model will be useful in next steps.

\section{ACKNOWLEDGMENTS}

The authors gratefully acknowledge the financial support by CAPES scholarships for stage postdoctoral, UFU, UFG/RC, FEMEC, FAPEG, FAPEMIG and by CNPq under MCTI-SECIS-CNPq-84-2013, proc. 458649/2013-9.

\section{REFERENCES}

Allin, S., Eckel, E., Markham, H., e Brewer, B. 2010. Recent trends in the development and evaluation of assistive robotic manipulation devices. Phys. Med. Rehabil. Clin. A. Am., 50, 59-77.

Baril, M., Laliberté, T., Gosselin, C., e Routhier, F. 2013. On the design of a mechanically programmable underactuated anthropomorphic prosthetic gripper. vol. 135.

Belter, J., Segil, J., Dollar, M., e Weir, R. 2013. Mechanical design and performance specifications of anthropomorphic prosthetic hands: A review. JRRD - Journal of Rehabilitation Research \& Development, 50(5), 599-618.

Craig, J. 2004. Introduction to Robotics - Mechanics and Control. USA: Prentice Hall. 3rd Edition.

Cutkosky, M. 1989. On grasp choice, grasp models, and the design of hands for macufacturing tasks. IEEE Transactions on Robotics and Automation, 5(3), 269-278. 
Dalley, S., Wiste, T., Withrow, T., e Goldfarb, M. 2009. Design of a multifunctional anthropomorphic prosthetic hand with extrinsic actuation. IEEE/ASME Transactions on Mechatronics, 14(6), 699-706.

Dechev, V., Cleghorn, W., e S., N. 2001. Multiple finger, passive adaptive grasp prosthetic hand. Mechanism and Machine Theory, 36, 1157-1173.

Du, H., e Charbon, E. 2007. 3d hand model fitting for virtual keyboard system.

Gaiser, I., Pylatiuk, C., Schulz, S., Kargov, A., Oberle, R., e Werner, T. 2009. The fluidhand iii: A multifunctional prosthetic hand. Journal of Prosthetics and Orthics, 21(2), 1-6.

Gosselin, C., Pelletier, F., e Laliberté, T. 2008. An anthropomorphic underactuated robotic hand with 15 dofs and a single actuator.

Ingram, J., Kording, K., Howard, I., e Wolpert, D. 2008. The statistics of natural hand movements. Exp. Brain Res., 188, 223-236.

Khalil, W., e Kleinfinger, J. 1986. A new geometric rotation for open closed-loop robots. IEEE International Conference on Robotics and Automation, 3(1), 1174-1179.

Langevin, G. 2014. Inmoov.

URL http: / /www.inmoov.fr/project/

Smagt, P., Grebenstein, M., Urbanek, H., Fligge, N., Strohmayr, M., e Stillfried, G. 2009. Robotics of human movements. Journal of Physiology, (103), 119-132.

Tarzimi, W., Elamvazuthi, I., e Begam, M. 2009. Kinematic and dynamic modeling of a multi-fingered robot hand. International Journal of Basics \& Applied Sciences, vol. 09, (pp. 61-68).

\section{RESPONSIBILITY NOTICE}

"The authors are the only responsible for the printed material included in this paper". 